\title{
A community outbreak of travel-acquired measles, Ontario 2009
}

\author{
Armstrong $\mathrm{J}^{1^{*}}$, Arajs $\mathrm{A}^{1}$ Bailey $\mathrm{N}^{1}$ and Wang $\mathrm{HL}^{1}$ \\ Region of Waterloo Public Health, Waterloo, ON \\ Corresponding author: JArmstrong@regionofwaterloo.ca
}

\section{Abstract}

Background: Canada has held elimination status for measles since 1998; however, imported cases continue to occur.

Objective: To describe the public health response to an imported measles case in the Waterloo Region of Ontario in May 2009.

Results: Contacts and exposures were traced, and cases were quickly investigated to identify the source. Through routine reporting mechanisms it was found that the index case had likely been exposed while on holiday in Disney World to a laboratory-confirmed measles case in a nine year old unimmunized boy from the United Kingdom (UK). Canada's National Microbiology Laboratory confirmed that the index case had the same D4 measles strain as the UK case and the strain that had been circulating in the UK. In total, one probable case and six confirmed cases were reported. The median age of confirmed cases was 14.5 years (mean age 17 years, range 6 to 39 years). Five confirmed cases (83\%) were female. One confirmed case (17\%) was hospitalized; no deaths were associated with the outbreak.

Conclusion: This outbreak highlights the importance of collaboration with clinical care, the laboratory and public health at all levels of government to investigate and control a measles outbreak. Global travel and sustained local transmission may continue to pose a challenge with respect to the eradication of measles in developed countries.

\section{Introduction}

Measles (rubeola) is an acute viral disease with a case fatality rate of 1-3 per 1,000 cases. Acute encephalitis occurs in approximately 1 of every 1,000 cases. Measles is one of the most highly communicable of all infectious diseases. It is a leading cause of vaccine-preventable deaths in children worldwide, the majority in developing countries (1, 2). Canada has held elimination status for measles since 1998. Nationally, sustained transmission has been eradicated by the current two-dose measles immunization programs and the high vaccine coverage in the general population; however, imported cases continue to occur (3).

On May 25, 2009, a local hospital reported a suspect case of measles in a 10 year old unimmunized female to Region of Waterloo Public Health. The case had presented to hospital, and measles was clinically suspected by an astute pediatrician. The serology result subsequently reported to Region of Waterloo Public Health on May 26, 2009, was positive (measles immunoglobulin M [IgM] reactive). Subsequent testing of urine samples and eye swabs by reverse transcriptase polymerase chain reaction (RT-PCR) detected measles virus ribonucleic acid (RNA), definitively confirming measles. The patient had travelled to Walt Disney World, Florida, from May 3 to 10, 2009.

This paper describes the local public health investigation, including identification of the source of infection, case definition, laboratory investigations, public health actions and risk communications. It highlights the importance of public health and infection control measures along with high community immunization rates to efficiently and effectively prevent further transmission and control outbreaks. 


\section{Methods}

\section{Source identification}

The index case was reported to the Ontario Agency of Health Protection and Promotion and the Ontario Ministry of Health and Long-Term Care in accordance with routine procedures.

\section{Case finding and data collection activities}

Cases were defined using the Ontario Ministry of Health and Long-Term Care criteria (Table 1) (4).

Table 1. Case definitions: community outbreak of travel-acquired measles, Waterloo Region, May-June 2009

\begin{tabular}{|c|c|}
\hline Case classification & Definition \\
\hline Confirmed & $\begin{array}{l}\text { Laboratory confirmation of infection with clinically compatible signs and symptoms in the } \\
\text { absence of recent immunization with measles-containing vaccine: } \\
\text { - Isolation of measles virus from an appropriate clinical specimen (e.g., nasopharyngeal } \\
\text { swab/aspirate/wash and urine); } \\
\text { - Detection of measles virus ribonucleic acid (RNA) from an appropriate clinical specimen; } \\
\text { - Seroconversion or a significant (e.g., fourfold or greater) rise in measles Immunoglobulin } \\
\text { G (IgG) titre by any standard serologic assay between acute and convalescent sera; } \\
\text { - Positive serologic test for measles Immunoglobulin M (IgM) antibody using a } \\
\text { recommended assay in a person who is either epidemiologically linked to a laboratory- } \\
\text { confirmed case or has recently travelled to an area of known measles activity; or } \\
\text { - Clinically compatible signs and symptoms in a person with an epidemiologic link (i.e., } \\
\text { close contact) to a laboratory-confirmed case. }\end{array}$ \\
\hline Probable & $\begin{array}{l}- \text { Clinically compatible signs and symptoms in the absence of appropriate laboratory tests } \\
\text { and in the absence of an epidemiologic link to a laboratory confirmed case; or } \\
\text { - Clinically compatible signs and symptoms in a person with recent travel to an area of } \\
\text { known measles activity. }\end{array}$ \\
\hline $\begin{array}{l}\text { Clinical evidence } \\
\text { criteria }\end{array}$ & $\begin{array}{l}\text { Clinically compatible signs and symptoms are characterized by all the following: } \\
\text { - Fever greater than or equal to } 38.3 \text { degrees Celsius (oral) and } \\
\text { - Cough, coryza or conjunctivitis (followed by) } \\
\text { - Generalized maculopapular rash for at least three days. }\end{array}$ \\
\hline
\end{tabular}

Cases were interviewed by Region of Waterloo Public Health nurses using a measles-specific questionnaire to document their clinical history, immunization history and other risk factors.

\section{Laboratory investigation}

Specimens were collected by the attending health care provider for each case. Laboratory testing was done at the Ontario Public Health Laboratory in Toronto, Ontario. Genotyping was conducted at the National Microbiology in Winnipeg, Manitoba.

\section{Public health actions}

Region of Waterloo Public Health initiated intense contact tracing for the index case by identifying all activities and exposures within the infectious period.

Regular communication with hospital infection control staff was maintained, and contact management of exposed patients was coordinated. Region of Waterloo Public Health managed community and physician office exposures, and the hospital infection control team managed hospital contacts. 


\section{Risk communication}

Communication messages for the public and for health care providers were coordinated by Region of Waterloo Public Health and shared in accordance with regular procedures.

\section{Results}

\section{Source identification}

The Ontario Ministry of Health and Long-Term Care shared outbreak information with other health units in Ontario and with the Public Health Agency of Canada (the Agency). Given that the index case had spent time in Florida during the exposure period, the Agency contacted the United States Centers for Disease Control and Prevention (CDC).

The CDC subsequently notified the Agency and Region of Waterloo Public Health of a laboratory-confirmed measles case in a nine year old unimmunized male from the United Kingdom (UK) who had visited Walt Disney World from May 1 to 15, 2009. Region of Waterloo Public Health compared the itineraries of the two cases and identified three occasions of possible contact. A timeline illustrating the likely transmission pathway that links the D4 measles strain circulating in the UK to the final measles case in the Waterloo Region outbreak is summarized in Figure 1.

Figure 1. Community outbreak of travel-acquired measles, Waterloo Region, May-June 2009

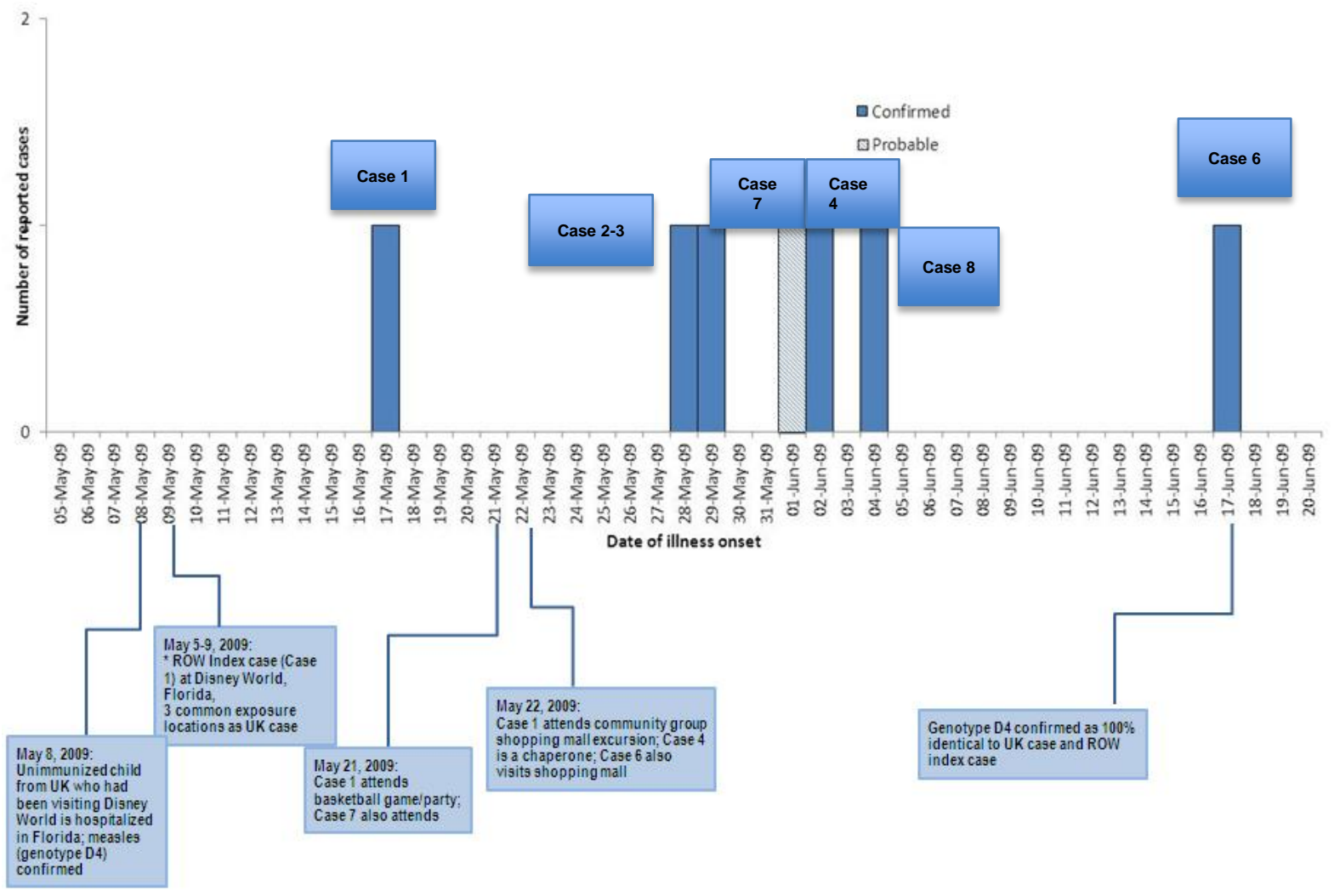

\footnotetext{
* ROW=Region of Waterloo
} 


\section{Descriptive epidemiology}

In total, one probable case and six confirmed cases were identified (Figure 1). The median age of confirmed cases was 14.5 years (mean age 17 years, range 6 to 39 years). Five confirmed cases (83\%) were female. One confirmed case (17\%) was hospitalized; no deaths were associated with the outbreak. A summary of the reported cases is given below.

\section{Cases 1-3}

The index case (Case 1) was an unimmunized 10 year old female who presented to hospital and whose disease was diagnosed by an astute pediatrician. She became quite ill and required hospitalization for several days, but no complications developed. Symptoms included a seven-day prodrome of cough and coryza followed by onset of intermittent fever, a maculopapular rash spreading from face to torso and bilateral conjunctivitis. On day four of the rash onset, measles IgM testing was done, which was reactive. Urine and eye swab RT-PCR testing was done on day 10 of symptom onset, both of which were positive; genotyping of the urine specimen was confirmed as identical to the D4 strain that had been circulating in the south of England since February 2009. A viral culture from the rash done on day 10 of symptom onset was negative. Two unimmunized siblings of the case, aged nine and six years old, showed measles symptoms 14 and 15 days after the index case onset respectively (Cases 2 and 3). These cases experienced a similar but milder course with no conjunctivitis and no requirement for hospitalization. No additional cases of measles were linked to these siblings.

\section{Case 4}

Case 4 was a 39 year old female (year of birth 1970) with a three-day prodrome of cough, chills and fever followed by a modified rash described as fine pink spots, which did not become confluent and which faded within two days. Case 4 chaperoned a mall excursion for a community group attended by Case 1 on May 22, 2009. Symptom onset was June 2, 2009, and measles was subsequently confirmed by serology. Immunization records indicated that Case 4 had received measles vaccine at age 11 months and measles mumps rubella vaccine (MMR) at five years of age. Case 4 was a nurse who cared for medically fragile children at a local hospital. She was advised to self-isolate and exclude herself from work; she remained off work until after the fourth day of rash onset. Measles IgM and IgG testing done on day five of rash onset were both reactive. Urine culture and PCR testing done on day eight of symptom onset were negative.

\section{Cases 5-6}

On June 24, 2009, a suspect case of measles in a 19 year old male was reported to Region of Waterloo Public Health. He had had no known direct contact with the index case. Case 5 was quite ill and sought medical attention in hospital; he was not admitted but was kept overnight in the emergency room. Symptom onset was June 17, 2009, and included a five-day prodrome of cough, coryza and fever followed by a maculopapular rash and conjunctivitis. Immunization records indicated that he had received two doses of MMR at nine and ten months of age in his country of origin. Measles IgM and IgG testing was done on day two of rash onset. Measles IgM was reactive; measles IgG was non-reactive. Convalescent measles IgG testing done on day 16 of symptom onset was reactive. Nasopharyngeal RT-PCR testing done on day eight of symptom onset was positive, and subsequent genotyping confirmed an identical match to the D4 strain of the index case.

During case management Case 5 indicated that his twin sister (Case 6) had shown similar symptoms on June 4, 2009, 13 days prior to onset of his own symptoms. Case 5 also indicated that his sister spent a lot of time at the local shopping mall associated with the outbreak. Subsequent investigation by Region of Waterloo Public Health determined that Case 6 had indeed been at the local shopping mall associated with the outbreak on May 22, 2009; this was confirmed with a transaction receipt for a purchase made that day. Measles was confirmed in Case 6 by measles IgM and IgG serology testing done one month after rash onset, both of which were reactive. Symptom onset included a five-day prodrome of cough, coryza and fever followed by a maculopapular rash. Immunization records indicated that she too had received two doses of MMR at 9 and 10 months of age in her country of origin. Case 6 was also quite ill and sought medical attention in hospital; she was not admitted but was kept overnight in the emergency room.

RT-PCR and genotyping from Case 5, the final case reported as part of the outbreak, identified the measles strain as D4, 100\% identical to the index case in Waterloo (Case 1). 


\section{Case 7 (probable case)}

Case 7 was an 11 year old female who showed symptoms 11 days after exposure to the index case at the basketball game/party on May 21, 2009. Symptoms were mild and included cough, coryza and sore throat followed by a low grade fever and rash. The rash spread from face to torso but was described as little bumps resolving in two days without becoming confluent. The case had received one dose of MMR vaccine at 13 months of age, at which time the parents decided to discontinue further immunization. Measles $\operatorname{lgM}$ and $\lg G$ testing was done on day five of the rash onset. The measles IgG was highly reactive, and the IgM was indeterminate. A throat swab for PCR and culture taken on day nine from symptom onset was negative. Further testing was declined by parents. Investigators speculated that this was a modified measles presentation, with minimal viral shedding due to partial immunization. Although this case did not technically meet the Ministry's guidelines ${ }^{(4)}$ for a probable case, it was classified as such, given the strong epidemiological link. An unimmunized sibling of Case 7 did not show symptoms.

\section{Laboratory investigation}

Genotyping of the Waterloo index case (Case 1) was confirmed to be a $100 \%$ identical match to the D4 strain identified in the UK case and the strain that had been circulating in the south of England since February 2009.

\section{Public health actions}

Exposures identified for the index case included hospital and community physician waiting rooms and numerous community activities. Two community activities were of particular interest: a basketball game/party on May 21 , 2009, and a community group excursion to a local shopping mall on May 22, 2009.

Active surveillance was initiated by means of direct telephone calls and e-mails to the approximately 87 identified community contacts at risk. One possible susceptible health care worker was identified in the community (Case 4). No susceptible health care workers were identified in the hospital's contact investigation. Immunization was discussed and encouraged with susceptible contacts or their parents; however, many were philosophically opposed to immunization. Susceptible contacts were advised to self-isolate until the end of the potential incubation period, and most were very cooperative. There were no high-risk contacts requiring immune globulin (i.e. immunocompromised people, infants, pregnant women).

\section{Risk communication}

Regular written advisories were faxed to all local primary health care providers and hospitals in Waterloo Region to provide case details, recommend increased surveillance, encourage immunization of susceptible individuals, avoid exposure to contacts in waiting rooms and report suspect cases. A media advisory was released to advise the public of the potential exposure date and time frame at the local shopping mall visited by the index case while infectious, and to encourage immunization for those susceptible. Letters and facts sheets were distributed to the families participating in the basketball program.

\section{Discussion}

This outbreak illustrates the highly infectious nature of measles and the potential for community-wide transmission from a single case. There was excellent collaboration among local, provincial and federal public health professionals and optimal coordination of public health and clinical care. Infection control practices in hospitals and physician offices likely played a role in preventing transmission. Case 1, Case 5 and Case 6 all spent time in hospital settings while infectious, yet no transmission occurred within the hospital. The high immunization rates in Waterloo Region (traditionally 90\%-95\% among school-age children from Englishspeaking publicly funded schools) were also a key factor in preventing further spread.

The six confirmed cases in this outbreak were either unimmunized or inadequately immunized. Case 4 and Case 7 were partially immunized and had milder illnesses and modified transient rashes. In both cases the PCR testing was negative, perhaps as a result of less viral shedding from partial immunization. This could explain the highly reactive measles $\lg G$ and no transmission to the unimmunized sibling of Case 7.

Case 5 and Case 6 had immigrated to Canada in 2005 and attended high school for several years in Ontario before moving to Waterloo Region to attend university. They had each received two MMR vaccinations in their country of origin, but at the ages of 9 and 10 months. This illustrates that those moving to Canada from 
developing countries may be inadequately immunized and may be a higher priority group. Immigrant populations are not required to demonstrate proof of immunization before entry to Canada and may be a particularly vulnerable group. Cases 5 and 6 were quite ill despite some immunization, perhaps because they had little or no protection. Serology testing for mumps and rubella demonstrated no immunity; both were supportive of immunization and subsequently received two doses of MMR vaccine in accordance with the Ontario immunization schedule.

Transaction purchase receipts are often used in foodborne outbreak investigations in an attempt to verify exposure to an implicated food item. Interestingly, this outbreak demonstrated the usefulness of a purchase receipt to epidemiologically link two previously unrelated cases and establish a connection between all of the reported cases in this outbreak.

\section{Conclusion}

This imported case of measles led to a relatively small outbreak of measles in Waterloo Region, given the highly infectious nature of the disease and an urban community of over 500,000 people. Factors that may have contributed to the limited spread within the community include a high rate of immunization, timely outbreak investigation, a high degree of compliance for self-isolation even in families philosophically opposed to immunization, risk communication and collaboration with local health care providers. Global travel may continue to pose a challenge with respect to the eradication of measles in developed countries, which reinforces the importance of maintaining high immunization rates in the community and continued vigilance for sporadic cases with the potential to cause outbreaks.

\section{Acknowledgements}

The authors would like to thank the following who assisted with managing and summarizing this outbreak: public health nursing staff from Region of Waterloo Public Health; Dr. J. Gubbay and Dr. R. Higgins and the laboratory technicians from the Ontario Agency of Health Protection and Promotion, Toronto, Ontario; the National Microbiology Laboratory in Winnipeg, Manitoba; Dr. A. Barron, pediatrician from Grand River Hospital, who astutely diagnosed the index case; Dr. W. Ciccotelli and the infection control practitioners from Grand River Hospital, Kitchener, Ontario; S. Dolman and the Ontario Ministry of Health and Long-Term Care, Toronto, Ontario; Public Health Agency of Canada, Ottawa, Ontario; Centers for Disease Control and Prevention, Atlanta, Georgia; Dr. L. Nolan, Region of Waterloo Public Health Medical Officer of Health; C. Harold, Region of Waterloo Public Health supervisor of information and planning; Jennifer Cutler, Region of Waterloo Public Health public health nurse.

\section{References}

(1) American Academy of Pediatrics Red Book 2009 Report of the Committee on Infectious Diseases. $28^{\text {th }}$ ed. Elk Grove Village, IL: American Academy of Pediatrics; 2009.

(2) Mandell G., Douglas R. and Bennett J. Principles and Practices of Infectious Disease $6^{\text {th }}$ ed. Philadelphia, PA: Elsevier Churchill Livingstone; 2005.

(3) Public Health Agency of Canada. Canadian Immunization Guide, 7th ed. Ottawa: Public Health Agency of Canda, 2006.

(4) Ontario Ministry of Health and Long-Term Care. Infectious Diseases Protocol, Toronto: Queen`s Printer for Ontario; 2009. 